\title{
Diagnostic adequacy and safety of endoscopic ultrasound-guided fine-needle aspiration in patients with lymphadenopathy in a large cohort
}

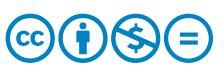

\author{
Authors \\ Gagandeep Kaur ${ }^{2}$, Haimanti Sarin ${ }^{2}$, Rajesh Puri ${ }^{1}$ \\ Institutions \\ 1 Institute of Digestive and Hepatobiliary Sciences, \\ Medanta The Medicity, Gurugram, India \\ 2 Department of Cytopathology, Medanta The Medicity, \\ Gurugram, India
}

Rinkesh K. Bansal', Narendra S. Choudhary', Saurabh K. Patle¹, Mahesh K. Gupta ${ }^{1}$, Chitranshu Vashishtha ${ }^{1}$,

submitted 31.7.2017

accepted after revision $\quad 20.10 .2017$

\author{
Bibliography \\ DOI https://doi.org/10.1055/s-0043-121986 | \\ Endoscopy International Open 2018; 06: E421-E424 \\ (c) Georg Thieme Verlag KG Stuttgart · New York \\ ISSN 2364-3722
}

Corresponding author

Rajesh Puri, MD Director, Institute of Digestive and Hepatobiliary Sciences, Medanta The Medicity, Sector 38, Gurgaon, Delhi NCR, India, PIN 122001

Fax: 01244834111

purirajesh70@gmail.com

\section{ABSTRACT}

Background and study aims The role of endoscopic-ultrasound (EUS) guided fine-needle aspiration (FNA) in patients with lymphadenopathy in terms of diagnostic ade- quacy and safety in large population is not well defined. The aim of this study was to evaluate diagnostic adequacy and safety of EUS-FNA in patients with lymphadenopathy. Patients and methods Retrospective study from October 2010 to September 2015 at tertiary care center in DelhiNCR. We analyzed data from 1005 EUS- FNAs of lymph nodes.

Results The study cohort comprised 1005 lymph nodes in 865 patients; $68 \%$ were males, mean age was $50 \pm 14$ years. Indications of FNA were to look for etiology of pyrexia of unknown origin or staging of malignancy mainly. FNA was taken from mediastinal nodes $(n=528,52.5 \%)$ and intra-abdominal nodes ( $n=477,47.5 \%$ ). Median size of nodes at long axis and short axis was $17(12-25.7)$ and $10(8-15)$ $\mathrm{mm}$ respectively. Adequate material by FNA was obtained in $92.8 \%$ cases. The cytopathologic diagnosis were malignancy in 153 (15.2\%), granulomatous change in 452 (42\%), and reactive lymphadenopathy in 328 (35.6\%). There was statistically significant difference seen between groups with pathological and reactive lymph nodes regarding size at long and short axis, hypoechoic nature, well defined borders and presence of necrosis and calcification. Procedure-related adverse effects were encountered in 6 patients $(0.8 \%)$. Four patients had mild mucosal bleeding in chronic liver disease patients and two had mild hepatic encephalopathy related to sedation.

Conclusion EUS-FNA of lymph nodes has good diagnostic adequacy and safety.

\section{Introduction}

The evolution of highly advanced diagnostic imaging modalities such as computer-aided tomography (CT) scanning, magnetic resonance imaging (MRI) and positron emission tomography (PET) has led to increasing detection of enlarged mediastinal and intra-abdominal lymph nodes. Most common indications for evaluating these lymph nodes were pyrexia of unknown origin (PUO) and malignancy. There are multiple causes of lymphadenopathy including benign conditions like tuberculosis and sarcoidosis. Malignant diseases like lymphoma and carcinoma also lead to lymph node enlargement which may be just reactive or secondary to involvement. These lymph nodes are difficult to FNA under ultrasound or CT guidance due to small size, unfavorable location or presence of vessels. Diagnostic laparoscopy, mediastinoscopy, and thoracoscopy are invasive and add unnecessary morbidity to a situation where the patient may have benign disease only.

Endoscopic ultrasound-guided fine-needle aspiration (EUSFNA) is a safe and effective method to obtain tissue diagnosis in patients with lymphadenopathy [1,2]. Endo-sonographic features alone predict malignant potential of lymph nodes, 
but fine needle aspiration cytology adds to diagnostic yield and accuracy [3-5]. EUS-FNA offers real-time vision along with proximity to target lesion and it has been proved to be very safe in presence of vascular structures with minimal complication rates and very good sensitivities and specificities $[6,7]$. There is lack of knowledge in term of diagnostic adequacy and safety in lymphadenopathy in large population and scarce data is available in the setting of pyrexia of unknown origin (PUO). The aim of the current study was to evaluate diagnostic adequacy and safety of EUS-FNA in patients with lymphadenopathy. In the present study, we describe our experience of EUSFNA of mediastinal or intra-abdominal lymph nodes in the setting of pyrexia of unknown origin or malignancy.

\section{Patients and methods}

The study was conducted from October 2010 to September 20152015 at a tertiary care center in North India (Delhi NCR). All patients who underwent EUS-FNA of lymph nodes were included in analysis. The study was conducted retrospectively after clearance from institute's review board/ethical committee. A total of 1005 EUS-FNAs of lymph noes were done at our center in the defined time period. Inclusion criteria were all patients $>18$ years with significant lymphadenopathy. EUS-FNA was done after obtaining informed consent. Exclusion criteria included age $<18$ years, pregnancy and uncorrected coagulopathy.

Anticoagulant and antiplatelet agents (clopidogrel) were withheld 5 days before the procedure (in 18 patients). Patients with platelet counts $<50,000$ and INR $>1.5$ received platelet concentrates and FFP transfusions, respectively. EUS-FNA was done for those nodes which were not easily accessible by percutaneous ultrasound and when diagnosis could not be achieved by other easier/noninvasive methods. All the procedures were carried out with conscious sedation (midazolam) or sometimes propofol given by the anesthesiologist. EUS-FNAs were done using GF-UCT180 curvilinear echo-endoscope (Olympus, Tokyo, Japan).

The primary objective of the study was to look for safety and diagnostic accuracy of EUS-FNA of lymph nodes. The following data were recorded for each patient; age, sex, indication for procedure, site of lymph node at short and long axis and ratio between them, echo features of lymph node (echogenicity, echo-structure, shape and border of lymph nodes, presence or absence of necrosis and calcification), size of needle and number of needle passes, number of slides, result of FNA, any procedural complications. When multiple lymph nodes were presence, nodes with larger size/having sharply demarcated borders/hypoechoic in nature were preferred for FNA. A lymph node was considered reactive when FNA showed lymphoid cells in different stages of activation in presence of adequate cellularity and absence of granulomas/necrosis/malignant cells.

A cytopathologist evaluated the material collected within 24 hours and a diagnosis was given. A specimen was considered adequate if there were an adequate number of representative cells from the lesion. If insufficient tissue was present to make a definitive diagnosis, further sampling was obtained via repeat
EUS-FNA. Diagnosis of tuberculosis (TB) was based on presence of caseating granulomas or acid-fast bacilli, and/or positive culture for Mycobacterium tuberculosis-positive gene Xpert in the EUS-FNA specimens.

Diagnosis of histoplasmosis was based on presence of caseating necrosis with granulomatous response. Gomori methenamine silver staining revealed abundant small intracellular budding yeast cells in macrophages where characteristic features of histoplasma were tiny 2 - to $4-\mu \mathrm{m}$ oval yeasts that reproduce by budding

\section{EUS-FNA procedure}

A lymph node was selected based on above mentioned criteria for FNA if feasible (avoidance of vascular structures and vital organs). A linear array echoendoscope was directly inserted into the esophagus. We used a 22-gauge needle in most cases (Cook Medical, Boston scientific). An EUS-FNA needle with stylet was introduced into the working channel; Doppler was used to avoid any vascular structures in needle path. The stylet was withdrawn slightly before puncture; it was reintroduced fully after puncture of the lymph node to displace any material in the needle (from gastrointestinal wall). After that stylet was completely withdrawn; 10 to 20 to-and-fro movements of the needle were done within the lymph node. The material inside the needle was pushed on the slides bit by bit with the help of the stylet. One or 2 slides from each pass were immediately fixed in absolute alcohol and the rest were air dried. The slides were stained with Papanicolaou, Giemsa stain and Ziehl-Neelsen stain (wherever required). The size of the needle and number of needle passes were operator-dependent.

\section{Statistical methods}

The data are shown as number, percentage and mean (SD) or median (IQR). Two groups were compared with Student's $t$ test or Mann-Whitney $\mathrm{U}$ test (for continuous variables) or Fisher's exact test or Chi-square test (for categorical data). A twotailed $P$ value $<0.05$ was considered significant. All statistical analysis was performed with SPSS, version 16 (SPSS, SPSS Inc., Chicago, IL).

\section{Results}

\section{Baseline characteristics}

The study was conducted retrospectively; it comprised EUSFNA of 1005 lymph nodes either mediastinal or intra-abdominal or both location in 865 patients; $68 \%$ males and $32 \%$ females. The mean age of the study group was $50 \pm 14$ years.

The primary indication for FNA was to look for pyrexia of unknown origin or unexplained weight loss in 680 patients (81.9\%), staging of malignancy in 118 patients (13.6\%); 67 (7.8\%) patients were asymptomatic and had significant lymphadenopathy (size of lymph node $>1 \mathrm{~cm}$ at short axis) which was detected incidentally on imaging studies.

FNA was taken from mediastinal nodes in $528(52.5 \%)$ individuals and from intra-abdominal nodes in 477 (47.5\%) individuals. The location of the targeted lymph nodes in the mediastinum included sub-carinal space in 410 cases $(40.8 \%)$, aorto- 
- Table 1 Comparison of pathologic and reactive lymph nodes in 933 aspirates.

\begin{tabular}{|l|l|l|l|}
\hline Parameter & $\begin{array}{l}\text { Pathological lymph nodes (n= 575) } \\
\text { (group A) }\end{array}$ & $\begin{array}{l}\text { Reactive lymph nodes (n=358) } \\
\text { (group B) }\end{array}$ & P value \\
\hline Age (years) & $49 \pm 15$ & $53 \pm 14$ & 0.27 \\
\hline Long axis (mm) & $20(15-28)$ & $15(12-20)$ & 0.000 \\
\hline Short axis (mm) & $12(9-15)$ & $9(7-12)$ & 0.000 \\
\hline Long/short axis ratio & $1.30(1.53-2.00)$ & $1.57(1.33-2.00)$ & 0.545 \\
\hline Number of slides & $11 \pm 3.304$ & $11.15 \pm 3.947$ & 0.966 \\
\hline Location (mediastinal/intra-abdominal) & $328 / 247$ & $162 / 196$ \\
\hline Echogenicity (hypo/hyper/mixed) & $416 / 52 / 107$ & $96 / 229 / 33$ & 0.000 \\
\hline Echo-structure (homo/heterogeneous) & $392 / 183$ & $293 / 65$ & 0.000 \\
\hline Necrosis (no/yes) & $440 / 135$ & $349 / 9$ \\
\hline Border (III-defined/well-defined) & $249 / 326$ & $108 / 250$ \\
\hline Calcification (no/yes) & $496 / 79$ & $344 / 14$ \\
\hline Number of passes & $2.2 \pm 1$ & $2.1 \pm 0.5$ & 0.000 \\
\hline
\end{tabular}

pulmonary window in 109 (10.8\%) and other mediastinal location in $9(0.9 \%)$. The location of lymph nodes in the abdomen comprised peri-portal in 184 cases (18.4\%), retroperitoneal in $166(16.5 \%)$ celiac axis in 106 cases (10.5\%) and peri-pancreatic in 21 cases $(2.1 \%)$.

Technical success (able to take FNA) was achieved in all cases. The median number of EUS-FNA passes was 2 (range 1 to 5). The 22 G EUS-FNA needle was used in the majority of lymph node FNAs $(n=968)$. Adequate material by FNA was obtained in $92.8 \%$ of cases. The cytopathologic diagnosis was malignancy in 153 (15.2\%), granulomatous change in 452 (42\%), and reactive lymphadenitis in 328 (35.6\%). A total of $7.2 \%$ FNAs were non-diagnostic (inadequate material). Of malignancies, 108 showed metastatic adenocarcinoma, 39 lymphoma and 6 showed neuroendocrine tumor. Patient baseline characteristics are summarized in $\mathbf{r}$ Table 1 . In patients with granulomatous material, there was either tuberculosis or sarcoidosis. In 344 patients, the diagnosis was tuberculosis (caseating granulomas 266, presence of acid-fast bacilli 55, positive culture for Mycobacterium tuberculosis 5 and positive gene Xpert 18). Histoplasmosis was diagnosed in 108 cases (caseating necrosis with granulomatous response with Gomori methenamine silver staining positivity).

When patients with pathological lymph nodes ( $n=575$, malignancy and granulomatous, group A) were compared to patients with non-pathological lymph nodes $(n=358$, reactive, group $B$ ) there was no significant difference regarding age and sex of patients, number of slides made, size of needle or number of needle passes. There was a significant difference between group $A$ and group $B$ in terms of location of lymph nodes $(P=0.000)$, mediastinal lymph nodes were significantly more pathological than abdominal lymph nodes.

Lymph node size was analyzed at the large and short axis and the ratio between large and short axis also analyzed. Median size of lymph nodes at the large axis was $20 \mathrm{~mm}$ (range 15 -
28 ) in group $A$ and $15 \mathrm{~mm}$ (range 12-20) in group B patients. Median size of lymph nodes at the short axis was $12 \mathrm{~mm}$ (range 9 -15) in group A while $9 \mathrm{~mm}$ (range $7-12$ ) in group B patients. Both sizes at the long axis and short axis were statistically significant $(P=0.000)$. There was no significant correlation found between the ratio of lymph node at the long axis with short axis and pathogenicity of lymph nodes $(P=0.545)$ as shown in - Table 1.

\section{EUS morphological features}

The echogenicity of lymph nodes was analyzed. Echogenicity were divided into 3 categories: hypoechoic, hyperechoic and mixed echoic. There was a significant correlation $(P=0.000)$ between echogenicity and pathogenicity of lymph nodes as shown in > Table 1.

Echo structure of lymph nodes was further analyzed. It was divided into 2 categories: homogeneous and heterogeneous. There was a significant correlation between echo-structure appearance of lymph nodes and their pathogenicity $(P=0.000)$.

Lymph node shape was analyzed. Shape was defined in $3 \mathrm{ca}-$ tegories: round, oval/triangular and oblong. There was a significant correlation between the shape of a lymph node and prediction of its pathogenicity $(P=0.000)$.

Borders were defined as ill-defined or well-defined. There was a significant association between well-defined border of lymph node and prediction of pathogenicity of lymph nodes $(P=0.000)$.

Any amount of calcification in a lymph node was analyzed. It was defined in 2 categories: present or absent. There was a significant correlation between lymph node calcification and prediction of pathogenicity of lymph nodes $(P=0.000)$.

Necrosis in lymph nodes was studied. It was defined in 2 categories: present or absent. There was a significant correlation between lymph node necrosis and prediction of pathogenicity of lymph nodes $(P=0.000)$. 


\section{Complications}

Immediate complications were recorded for all procedures during EUS and 2 hours after the procedure. Procedure-related adverse effects were encountered in 6 patients $(0.8 \%)$. Four patients with chronic liver disease had mild mucosal bleeding and two had mild hepatic encephalopathy related to sedation. No significant intra-procedural bleeding or bowel perforations after FNA were encountered and there was no significant correlation in both the groups.

\section{Discussion}

Our study is the largest study of lymph nodes examined with EUS-FNA in the published literature. The results of this study clearly demonstrate that EUS-FNA has a high rate of success in sampling tissue from enlarged lymph nodes whether mediastinal or intra-abdominal location. EUS-FNA has been established as a minimally invasive and safe method for diagnosing the cause of lymphadenopathy.

Almost three-quarters of the lymph nodes studied were benign, i. e. granulomatous or reactive. Fifteen percent of lymph nodes were malignant. A total of $7.2 \%$ were non-diagnostic. In a study of EUS-FNA of intra-abdominal lymph nodes in patients, $63 \%$ of patients had malignancy while benign disease was diagnosed in $37 \%$ of patients [8]. The Relatively high prevalence of granulomatous disease in tropical countries like India may be the best explanation.

Multiple series published on mediastinal lymph nodes have reported a diagnosis rate of up to $90 \%$, with a sensitivity and specificity of $85 \%$ and $100 \%$, respectively $[2,9,10]$. In a study of intra-abdominal lymph nodes, sensitivity was $89.7 \%$ and specificity was $98.3 \%$ [8]. In a study for utility of EUS-FNA in diagnosis of mediastinal and intra-abdominal lymphomas, sensitivity was $96 \%$ and specificity was $100 \%$ with a complication rate of only $1 \%$ [11]. Our study did not measure sensitivity or specificity but it did show a diagnostic adequacy of sample tissue with $92.8 \%$ success.

Size at the long and short axes were significantly associated with pathological and reactive nodes. When we compared echo features of lymph nodes including echogenicity, echo structure, shape and border of lymph nodes, presence of necrosis and calcification and calcification, they were significantly different in pathological and reactive lymph nodes. In a different series of 33 patients with small lymph nodes, there was no significant difference between pathologic (malignant and granulomatous) and reactive lymph nodes regarding size at the long and short axes, ratio of long and short axis, hypoechoic nature and sharply defined borders [12]. Because this study had a large number of patients, pathological features seemed to play a significant role along with cytopathological features in differentiating between pathological and reactive lymph nodes.

Our study had certain limitations. First, it was retrospective. Second, we did not compare our diagnostic yield with surgical or clinical data. Third, we do not have follow-up data. Fourth, sensitivity, specificity and diagnostic accuracy of EUS-FNA cannot be calculated for this study cohort as the gold standard of diagnosis (excision pathology of lymph nodes) is not available in the majority of patients. Excision biopsy of visceral lymph nodes carries risk of complication and it is unethical to perform it if EUS-FNA provides some diagnostic information.

\section{Conclusion}

EUS-FNA is a reliable, safe, highly effective and minimally invasive method to establish a diagnosis in patients with lymphadenopathy.

\section{Acknowledgements}

Data collection was done by Dr. Sweety Bansal, Mr. Yogesh Saini, Miss Babra Javaid, Miss Himanshi Saini (research coordinators) and Mr. Manish K. Singh (statistician).

\section{Competing interests}

\section{None}

References

[1] Catalano MF, Sivak MV Jr, Rice T et al. Endosonographic features predictive of lymph node metastasis. Gastrointest Endosc 1994; 40: $442-446$

[2] Williams DB, Sahai AV, Aabakken L et al. Endoscopic ultrasound guided fine needle aspiration biopsy: a large single centre experience. Gut 1999; 44: $720-726$

[3] Chen VK, Eloubeidi MA. Endoscopic ultrasound-guided fine needle aspiration is superior to lymph node echo features: A prospective evaluation of mediastinal and peri-intestinal lymphadenopathy. Am J Gastroenterol 2004; 99: 628-633

[4] Nakahara O, Yamao K, Bhatia V et al. Usefulness of endoscopic ultrasound-guided fine needle aspiration (EUS-FNA) for undiagnosed intra-abdominal lymphadenopathy. J Gastroenterol 2009; 44: 562 - 567

[5] Bhutani MS, Hawes RH, Hoffman BJ. A comparison of the accuracy of echo features during endoscopic ultrasound (EUS) and EUS-guided fine needle aspiration for diagnosis of malignant lymph node invasion. Gastrointest Endosc 1997; 45: 474-479

[6] Puli SR, Batapati KrishnaReddy J et al. Endoscopic ultrasound: it's accuracy in evaluating mediastinal lymphadenopathy? A meta-analysis and systematic review World journal of gastroenterology: WJG 2008; 14: $193028-3037$

[7] Chhieng DC, Jhala D, Jhala $N$ et al. Endoscopic ultrasound guided fine needle aspiration biopsy: A study of 103 cases. Cancer 2002; 96: $232-239$

[8] Korenblit J, Anantharaman A, Loren DE et al. The role of endoscopic ultrasound-guided fine needle aspiration (eus-fna) for the diagnosis of intra-abdominal lymphadenopathy of unknown origin. J Interv Gastroenterol 2012; 2: 172-176

[9] Catalano MF, Alcocer E, Chak A et al. Evaluation of metastatic celiac axis lymph nodes in patients with esophageal carcinoma: accuracy of EUS. Gastrointest Endosc 1999; 50: 352-356

[10] Catalano MF, Nayar R, Gress F et al. EUS guided fine needle aspiration in mediastinal lymphadenopathy of unknown etiology. Gastrointest Endosc 2002; 55: 863-911

[11] Yasuda I, Tsurumi H, Omar S et al. Endoscopic ultrasound guided fine needle aspiration biopsy for lymphadenopathy of unknown origin. Endoscopy 2006; 38: 919-924

[12] Choudhary NS, Bansal RK, Puri R et al. Impact of endoscopic ultrasound-guided fine needle aspiration of small lymph nodes. J Dig Endosc 2016; 7: 51 - 54 